\title{
Can we quickly and thoroughly assess pain with the PACSLAC-II? A convergent validity study in long-term care residents suffering from dementia
}

Mélanie Ruest, OT, ${ }^{1,2}$ Monique Bourque, RN, ${ }^{4}$ Sarah Laroche, ${ }^{1}$ Marie-Philippe Harvey, ${ }^{2}$ Marylie Martel, ${ }^{2}$ Kayla Bergeron-Vézina, ${ }^{2}$ Catherine Apinis, PT, MSc, ${ }^{1,3}$ Dominique Proulx, RN, ${ }^{4}$ Thomas Hadjistavropoulos, PhD, ${ }^{5}$ Yannick Tousignant-Laflamme, PT, $\mathrm{PhD},{ }^{1,3}$ Guillaume Léonard, $\mathrm{PT}, \mathrm{PhD},{ }^{1,2}$

${ }^{1}$ School of Rehabilitation, Faculty of Medicine and Health Sciences, Université de Sherbrooke, Sherbrooke, Quebec, Canada; ${ }^{2}$ Research Centre on Aging, Centre intégré universitaire de santé et de services sociaux de l'Estrie (CIUSSS de l'Estrie-CHUS), Sherbrooke, Quebec, Canada; ${ }^{3}$ Centre de recherche du Centre Hospitalier Universitaire de Sherbrooke (CRCHUS), Sherbrooke, Quebec, Canada; ${ }^{4}$ Centre intégré universitaire de santé et de services sociaux de l'Estrie - Centre hospitalier universitaire de Sherbrooke (CIUSSS de l'Estrie-CHUS), Sherbrooke, Quebec, Canada; ${ }^{5}$ Department of Psychology and Centre on Aging and Health, University of Regina, Regina, Saskatchewan, Canada

Corresponding author: Guillaume Léonard, PT, Ph.D. Research Centre on Aging, CIUSSS de l'Estrie - CHUS 1036, rue Belvédère Sud Sherbrooke (Québec) J1H 4C4

Telephone: 819-829-7131 ext. 45246

Email: guillaume.leonard2@usherbrooke.ca 


\section{ABSTRACT}

Background: A previous study showed that the modified version of the Pain Assessment Checklist for Seniors with Limited Ability to Communicate (PACSLAC-II) is a valid tool to assess pain in elderly individuals suffering from dementia and who are unable to communicate verbally.

Aims: The primary objective of this study was to confirm the convergent validity of the PACSLAC-II using direct evaluation of long-term care (LTC) residents in real-life situations, using two other well validated pain assessment scales (i.e. PACSLAC and Pain in Advanced Dementia [PAINAD]). A secondary objective was to document and compare the time required to complete and score each assessment scale.

Methods: Forty-six LTC residents (mean age $=83 \pm 10$ years) suffering from dementia were observed during two potentially painful procedures (transfer/mobilization), by three independent evaluators, each using one of the assessment scales (randomly assigned). Correlational analyses and analysis of variance were used to evaluate the association between each scale and to compare scoring time.

Results: The PACSLAC $(r=0.61)$ and the PAINAD $(r=0.65)$ were both moderately associated with the PACSLAC-II (all p-values < 0.001). The PAINAD's average scoring time $(63 \mathrm{~s} \pm 19 \mathrm{~s})$ was lower than the PACSLAC-II's $(96 \mathrm{~s} \pm 2 \mathrm{~s})$, which was lower than the PACSLAC's $(135 \mathrm{~s} \pm 53 \mathrm{~s})$ (all p-values $<0.001)$.

Conclusion: These results suggest that the PACSLAC-II is a valid tool for assessing pain in individuals with dementia. The time required to complete and score the PASCLAC-II was reasonable, supporting its usefulness in clinical settings. 
Keywords: pain, long-term care, nursing home, elderly, dementia, Alzheimer's disease 


\section{BACKGROUND}

Chronic pain is a significant health problem among older adults living in long-term care (LTC) (Takai, Yamamoto-Mitani, Okamoto, Koyama, \& Honda, 2010). Although chronic pain is not life-threatening, its consequences on mood, physical function, autonomy, sleep, and quality of life are significant (Chen, Hayman, Shmerling, Bean, \& Leveille, 2011; Fine, 2011; Greenberg, 2012; Leveille, Bean, Ngo, McMullen, \& Guralnik, 2007; Muller, Thomas, \& Peat, 2012; Stubbs, Patchay, Soundy, \& Schofield, 2014). For instance, in a cohort of community-dwelling women aged 65 years and over, Leveille and colleagues showed that lower extremity pain increased the likelihood that the individual would have difficulty with stair climbing (Leveille, Bean, Ngo, McMullen, \& Guralnik, 2007). Similar results were obtained by Muller and colleagues, who observed that the onset of lower limb pain, in a population of adults aged over 50 years, was associated with increased locomotor disability (Muller, Thomas, \& Peat, 2012).

Dementia is an important health problem affecting almost 50 million people worldwide (OMS, 2012). Like chronic pain, the prevalence of dementia substantially increases with age (Katz et al., 2012; Mathillas, Lovheim, \& Gustafson, 2011; Scherder et al., 2009). Given that age is a common risk factor for both chronic pain and dementia, many patients suffering from dementia also suffer from chronic pain (Scherder et al., 2005). The communication problems encountered in individuals with dementia make the evaluation of pain in these patients especially challenging for healthcare providers. The difficulty with pain assessment probably contributes to the under evaluation, under estimation and under treatment of pain reported for this population (Shega, Hougham, Stocking, Cox-Hayley, \& Greg, 2004; Zwakhalen, Hamers, \& Berger, 2006b). Indeed, 
several studies have shown that people with dementia are less likely to receive adequate analgesic treatment, compared to people who can verbalize and describe their pain (Shega, Hougham, Stocking, Cox-Hayley, \& Greg, 2004; Zwakhalen, Hamers, AbuSaad, \& Berger, 2006a; Zwakhalen, Hamers, \& Berger, 2006b). These findings are particularly worrisome, considering the observations of Kunz et al. (2009) and JensenDahm et al., (2014), who noted evidence of intensified processing of noxious information in some people suffering from dementia.

To overcome the communication barriers associated with dementia and to facilitate the evaluation of pain in individuals presenting dementia, several assessment tools were developed over the last few years (Storti et al., 2014; Zwakhalen, Hamers, Abu-Saad, \& Berger, 2006a). In a past review, Zwakhalen et al. (2006a) identified 12 observational pain assessment scales that can be used to assess pain in elderly individuals with severe dementia. The authors concluded that three scales: i) the Pain Assessment Checklist for Seniors with Limited Ability to Communicate (PACSLAC) (Fuchs-Lacelle \& Hadjistavropoulos, 2004), ii) the Pain Assessment in Advanced Dementia (PAINAD) (Warden, Hurley, \& Volicer, 2003), and iii) the DOLOPLUS-II (Wary, Serbouti, \& Doloplus, 2001) were particularly commendable. Another expert consensus showed that the PACSLAC and the PAINAD were among the most relevant tools, considering their metrological qualities (Herr, Bursch, Ersek, Miller, \& Swafford, 2010). Although these reviews do not allow to identify the ideal pain assessment tool (Kaasalainen, AkhtarDanesh, Hadjistavropoulos, Zwakhalen, \& Verreault, 2013), accumulating data suggest that the PACSLAC could be an excellent choice for the health care professionals working 
in a nursing home context (Hadjistavropoulos et al., 2014; Herr, Bursch, Ersek, Miller, \& Swafford, 2010; Zwakhalen, Hamers, Abu-Saad, \& Berger, 2006a).

Despite its excellent psychometric qualities, the PACSLAC is rarely used by healthcare providers, possibly because it is one of the longest tools of its kind, consisting of 60 items. This situation encouraged Chan and colleagues (2014) to develop a shorter version of the PACSLAC, improving the validity of the original version and reducing the number of items to assess. They noted that the new version of the PACSLAC, the PACSLAC-II, containing 31 rather than 60 items, had satisfactory reliability, excellent validity, and could successfully differentiate individuals in pain and in non-pain states. The authors observed that the PACSLAC-II was highly correlated with the PACSLAC and with the PAINAD. It is also worth noting that the PACSLAC-II retained coverage of all the pain assessment domains recommended by the American Geriatrics Society (AGS) for the assessment of pain in the nonverbal adult (AGS Panel, 2002).

The results obtained by Chan and colleagues (2014) were based on the evaluation of pain behaviors which was assessed by research assistants using pre-recorded video clips of LTC residents. Although valuable, these results need to be replicated in real clinical settings (i.e., direct evaluation in LTC facilities) before any final conclusions can be made. Accordingly, the aim of the present study was to confirm the convergent validity of the PACSLAC-II using direct evaluations of LTC residents in real-life situations. More specifically, the primary objective was to assess the relationships between the PASCLAC-II and the PACSLAC, as well as between the PACSLAC-II and the PAINAD. A secondary objective was to document and compare the time required to complete and score each assessment scale. We hypothesized that: 1) both the PACSLAC 
and the PAINAD would be highly correlated with the PACSLAC-II, and 2) scoring time for the PACSLAC would be longer than for the PACSLAC-II, which would be longer than for the PAINAD (which contain only 5 items).

\section{METHODS}

\section{Research design}

This study used a correlational framework to initiate the study of convergent validity between the scores of the PACSLAC-II with those obtained from two other pain assessment scales. Data were collected at one point in time in the clinical settings by three different evaluators.

ParticipantsParticipants were recruited in LTC centers in Southeastern Canada using convenience sampling. Participants with dementia were identified by consulting the medical records of every resident of both hospitals and residential centers. Participants were included in the study if they were aged 50 years or older, had received a diagnosis of dementia and had a score of -2 or -3 on the last item of the "Communication" section of the Functional Autonomy Measurement System - Système de mesure de l'autonomie fonctionnelle (SMAF) (Hébert et al., 2003). This last criterion ensured that the individuals included had serious communication difficulties (unable to answer simple questions requiring "yes" or "no" answers). Participants who were unable to be transferred or moved safely by the nursing staff (e.g., a patient with a recent lower limb facture) were excluded for safety reasons.

One hundred and ten (110) participants met the eligibility criteria (see Figure 1). Invitation letters were sent by mail to each potential participant's legal representative 
who was later contacted by telephone by a research assistant to determine if they had any questions about the project. Consent was obtained from the legal representatives for 46 LTC residents. Upon receipt of the signed consent letters, the research assistant called the LTC nursing staff to schedule the evaluation, based on each resident's normal routine. The 46 LTC residents for which consent was obtained were assessed. For the residents with whom it was possible to communicate briefly, their assent was obtained prior to the evaluation.

Insert Figure 1 here

\section{Assessment tools}

The pain behaviors were assessed using the PACSLAC, the PACSLAC-II and the PAINAD scales. The specifications and psychometric properties of each tool are briefly summarized below.

PACSLAC. The PACSLAC is a 60-item assessment scale that was developed by FuchsLacelle and Hadjistavropoulos (2004). The 60 items are divided into 4 sections: facial expressions, activities and movement, behavior/personality/mood, and other (physiological changes, changes in eating or sleeping, and vocal behaviors). Each item is scored as present (1) or absent (0). The item scores are then added with higher scores being indicative of higher levels of pain. The clinical usefulness and psychometric properties of the PASCLAC are well established (Aubin et al., 2008; Cheung \& Choi, 2008; Herr, Decker, \& Bjoro, 2004; Lints-Martindale, Hadjistavropoulos, Lix, \& Thrope, 2012; Zwakhalen, Hamers, \& Berger, 2006b). Considering the time spent by the evaluators with the LTC residents and the task observed (transfer/mobilization), items related to eating or sleeping were not evaluated. 
PACSLAC-II. The PACSLAC-II is a 31-item assessment scale which was recently developed by Chan et al. (2014). The PACSLAC-II items were designed to include behaviors that had been shown to discriminate painful and non-painful states while minimizing overlap with behaviors that also occur in non-painful situations such as delirium. As it is the case in the PACSLAC, the total score of the PACSLAC-II corresponds to the sum of the checked items. The PACSLAC-II items are organized per the six pain assessment domains that have been recommended by the AGS (AGS Panel, 2002). In their study, Chan et al. (2014) found that the PACSLAC-II had good psychometric qualities (satisfactory internal consistency and interrater reliability, and good convergent and discriminant validity).

PAINAD. The PAINAD was developed by Warden et al. (2003) by adapting the Discomfort Scale for patients with Dementia of the Alzheimer Type (DS-DAT) scale (Hurley, Volicer, Hanrahan, Houde, \& Volicer, 1992). This simple and brief assessment scale contains 5 items: breathing, negative vocalization, facial expression, body language and consolability (ability to be comforted) that are rated between 0 (no sign of pain) and 2 (presence of signs of pain) for a total of 10 points. Zwakhalen et al. (2012) reported that a score of 2 or more suggests the presence of pain. The validity and reliability of the PAINAD are well established (Warden, Hurley, \& Volicer, 2003; Zwakhalen, Hamers, \& Berger, 2006b). Contrary to the PACSLAC and the PACSLAC-II (which both cover the six recommended domains of nonverbal behaviors proposed by the AGS: facial expressions, verbalizations/vocalizations, body movements, changes in interpersonal interactions, changes in activity patterns or routines, and mental status changes; AGS 
Panel, 2002), the PAINAD covers only three domains (i.e., facial expressions, verbalizations/vocalizations, and body movements; Herr, Bjoro, \& Decker, 2006).

\section{Procedures and ethical considerations}

Medical files for each patient were retrieved to confirm participant eligibility and to document all other relevant information. This included the patient's sociodemographic information (e.g., age, gender), medical diagnosis, level of cognitive impairment (as assessed by the SMAF) and medical history related to pain (number and types of painful pathologies associated with their medication). As specified earlier, the consent was obtained through the signed letters from the legal representatives for all participants (i.e., LTC residents) recruited.

The pain assessment procedures took place in each participant's room from July 2013 to March 2014. Three independent evaluators from the research group (research assistants) simultaneously observed the patient during a usual transfer (e.g., transfer from bed to chair) or mobilization (e.g., change of position in bed), two potentially painful procedures. The assessment forms were completed immediately after the observation, outside the patient's room. No discussions occurred between the raters during the completion of the assessment scales. Each evaluator used a standard chronometer (stopwatch) to record the time required to complete the assessment forms. The evaluators were randomly assigned (using a random numbers table) to one of the three assessment scales selected in this study (PACSLAC-II, PACSLAC and PAINAD) for each participant. Before the beginning of data collection, all the evaluators underwent specific training on the use of the three assessment scales to ensure appropriate scoring and standardization. 
The information, obtained from the medical patients' charts and from the assessment scales, was stored on a secure computer at the Research Centre on Aging of the CIUSSS de l'Estrie - CHUS. A coding system was used to ensure information confidentiality. The Research Ethics Board of the CIUSSS de l'Estrie-CHUS approved the study's procedures.

\section{Data analysis}

To assess the convergent validity of the PACSLAC-II (objective 1), correlational analyses (Pearson's Rho) were used to quantify the relationships between the PACSLACII and the PACSLAC and between the PACSLAC-II and the PAINAD. Pearson coefficients were considered as evidence of negligible (between 0 and 0.3 ), low (between 0.31 and 0.5 ), moderate (between 0.51 and 0.7 ), high (between 0.71 and 0.9 ) and very high (between 0.91 and 1) associations, respectively (Hinkle et al., 2003; Mukaka, 2012). A repeated-measures Analysis of Variance (ANOVA) was used to compare scoring times between the three assessment scales (objective 2). Results were considered to be significant if $\mathrm{p}<0.05$ was obtained. All tests were performed using SPSS $®$ (version 17.0 for Windows ${ }^{\circledR}$, Chicago, IL, USA).

\section{RESULTS}

\section{Participants}

Forty-six (46) LTC residents (36 women and 10 men) aged between 52 and 96 years old (mean age $83 \pm 10$ years) participated in the study. The characteristics of the residents are presented in Table 1. Every resident had a diagnosis of dementia. Per the medical records, most of the 46 LTC residents were affected by a potentially painful health 
condition and received prescribed analgesic medication on a regular basis. All the participants needed partial or total help from orderlies to complete their transfers or mobilizations.

\section{Insert Table 1 here}

Convergent validity

The mean scores and standard deviations obtained with the PACSLAC-II, the PACSLAC and the PAINAD were $5.9 \pm 3.2,7.0 \pm 4.0$ and $3.3 \pm 2.0$, respectively. Correlational analysis showed that there was a moderate relationship between the score from the PACSLAC-II and the score from the PACSLAC $(r=0.61 ; p<0.001)$, as well as between the score from the PACSLAC-II and the score from the PAINAD $(r=0.65 ; p<$ 0.001) (see Table 2).

Insert Table 2 here Scoring times

Scoring times for the three assessment scales are shown in Figure 2. Scoring time for the PACSLAC was higher than for the PACSLAC-II, which was higher than for the PAINAD. These differences were confirmed by the repeated-measure ANOVA and by the post-hoc paired sample t-tests, which revealed that the scoring time for the PACSLAC-II was significantly lower than for the PACSLAC, and significantly higher than for the PAINAD (all p-values < 0.001).

Insert Figure 2 here

\section{DISCUSSION}


The objectives of the present study were to confirm the convergent validity of the PACSLAC-II by using real-time patient evaluations and to document and compare the scoring time of the PACSLAC-II with that of the PACSLAC and the PAINAD. We observed that: i) the PACSLAC-II was moderately correlated with the PACSLAC and with the PAINAD, and ii) the scoring time from the PACSLAC-II was lower than the PACSLAC and higher than the PAINAD. These results represent a new step in the establishment of convergent validity for the PACSLAC-II.

Convergent validity of the PACSLAC-II

The development and initial validation of the PACSLAC-II was completed by Chan and colleagues (2014). In their study, these authors assessed the relationship between the PACSLAC-II and 6 other behavioral pain assessment scales (including the PACSLAC and the PAINAD) in 124 LTC residents during one non-painful procedure (i.e., swabbing) and 2 potentially painful procedures (i.e., vaccination and movement). They reported significant correlations between the PACSLAC-II and the PACSLAC and between the PACSLAC-II and the PAINAD for both non-painful (all r-values $\geq 0.66$ ) and painful conditions (all r-values $\geq 0.79$ ). The identified correlation coefficients in the present study $(r=0.61$ and 0.65$)$ are like those reported by Chan and colleagues (2014). Contrary to Chan and colleagues, who used pre-recorded video footage, the assessments for this study were performed directly in the LTC residents' rooms (live evaluations). Our results are congruent with the results obtained by Chan and colleagues (2014) and confirm that the PACSLAC-II is a valid and appropriate pain assessment scale for seniors with limited ability to communicate. 
During the development and initial validation of the original version of the PACSLAC, Fuchs-Lacelle and Hadjistavropoulos (2004) noted that the PACSLAC demonstrated excellent psychometric properties, including high levels of internal consistency (Cronbach's $\alpha=0.82-0.92$ ), as well as the capacity to discriminate between painful and non-painful events (Fuchs-Lacelle \& Hadjistavropoulos, 2004). Slightly lower Cronbach's $\alpha$ were reported by Liu and colleagues (2010) in individuals with and without cognitive problems suffering from osteoarthritis $(\alpha=0.70-0.79)$. These authors also noted that the PACSLAC had good inter-rater reliability, with intra-class correlation coefficients oscillating between 0.68 and 0.82 . Just like the PASCLAC, the PACSLAC-II was reported to have excellent psychometric qualities and was also able to discriminate between painful and non-painful states (Chan, Hadjistavropoulos, Williams, \& LintsMartindale, 2014). In the present study, the reliability of the PASCLAC-II (internal consistency, intra and inter-rater reliability) was not assessed. Future studies, considering the reliability of the PACSLAC-II using real-time evaluations in LTC facilities, should be performed to further document the psychometric qualities of the PACSLAC-II and confirm its utility in a clinical setting.

\section{Scoring time}

Consistent with our initial hypothesis, we observed that completion times for the PACSLAC-II were shorter than for the PACSLAC, but longer than for the PAINAD. Although statistically significant, the difference was not substantial. For example, we observed a mean difference of 33 seconds between the PACSLAC-II scoring time and the PAINAD scoring time. The small difference observed between the scoring times for these two pain assessment scales can be surprising when we consider that the PASCLAC- 
II has six times more items than the PAINAD. This situation could, paradoxically, be explained by the brevity of the PAINAD. Indeed, the evaluators often reported being hesitant when scoring certain items from the PAINAD because of their relative weights on the total score to be obtained. Contrary to the PACSLAC-II - for which each item accounts for a small portion of the total score (i.e., 3\%) - each item of the PAINAD accounts for $20 \%$ of the total score. This situation often led the evaluators to be more hesitant when scoring certain items from the PAINAD, particularly when the behavior assessed was not obviously present or absent. This is consistent with the observations of Zwakhalen et al. (2006b), who reported that nurses using the PAINAD commented about the negative impact of the briefness of this questionnaire. Finding a good balance between too many items and too few items to evaluate is certainly challenging. With its 31 items, we believe that the PACSLAC-II has succeeded in addressing this challenge. The context of care can probably have a significant influence on the most appropriate and preferred scale. In a certain milieu, such as a short-term geriatric unit, perhaps the PAINAD would be more suitable, whereas the PACSLAC-II could be more useful in LTC homes.

\section{Limitations}

Some important limitations must be acknowledged for this study. First, the sample size of the participants is relatively small $(n=46)$. Second, although the three evaluators were first given training to standardize their methods of administration for each tool, none of them had prior clinical experience with the pain assessment scales. Finally, no follow-up was made during the study with the evaluators to ensure that the assessment tools were used appropriately and in a consistent manner. 


\section{Implications for nursing education practice and research}

Implications for practice. Nursing care of elderly individuals suffering from dementia requires a holistic approach to ensure proper management of the factors that influence their well-being (Cavalieri, 2005). Effective pain management improves patient quality of life. Given its brevity and ease of administration, the PACSLAC-II has the potential of supporting nurses in their assessment and treatment of pain. The validation of the PACSLAC-II as well as of other tools of this kind contributes to the advancement of nursing care. The use of a tool like the PACSLAC-II will allow nurses to improve their practice by helping them document pain-related information in a standardized fashion. Standardized reporting of pain levels in clinical charts facilitates communication with other healthcare professionals, for the benefit of LTC residents. Implications for research. Research on the development and validation of pain assessment tools for elderly individuals with dementia has markedly increased in the past years. The PACSLAC is considered by many researchers as being one of the most valid pain assessment tools (Ellis-Smith et al., 2016). Validation of the PACSLAC-II (the short and improved version of the PACSLAC) in real-life situations will contribute to research in nursing. Future studies should examine further the sensitivity of the PACSLAC-II in detecting fluctuations in pain (e.g., following the administration of analgesic medications). Such research will facilitate the development of a systematic approach to the use of observational pain assessment scales in LTC, which would be especially important given that people with advanced dementia are less likely to undergo adequate pain treatment (Liu \& Leung, 2016). 


\section{CONCLUSION}

In the past few years, many pain assessment tools were developed to evaluate pain in older individuals suffering from dementia. This study confirms that the PACSLAC-II is a valuable assessment scale that can be used to evaluate pain in LTC residents suffering from dementia. The PASCLAC-II has good psychometric qualities and can be scored quickly which make it suitable for healthcare professionals working in LTC settings.

\section{Conflict of interest}

Thomas Hadjistavropoulos is one of the developers of the PACSLAC and the PACLSAC-II but has no commercial interests regarding these two tools. The other authors have no conflict of interest to report.

\section{Acknowledgments}

The authors wish to thank the CIUSSS de l'Estrie-CHUS staff members for their help with data collection, as well as all the patients who participated in this project. Guillaume Léonard is supported by FRQ-S (Québec).

\section{Funding}

This work was supported by a grant from the CIUSSS de l'Estrie-CHUS Vitae Foundation. 


\section{REFERENCES}

American Geriatrics Society [AGS] Panel on Persistent Pain in Older Persons. (2002).

The management of persistent pain in older persons. American Geriatrics Society, $50, \mathrm{~s} 205-\mathrm{s} 224$.

Aubin, M., Verreault, R., Savoie, M., LeMay, S., Hadjistavropoulos, T., Fillion, L., Beaulieu, M., Viens, C., Bergeron, R., Vézina, L., Misson, L., \& Fuchs-Lacelle, S. (2008). Validity 'and Utilities' clinic of a grid observation (PACSLAC-F) to evaluate the pain in seniors with dementia's living in the Long-Term Care. Canadian Journal on Aging, 27, 45-55.

Cavalieri, T. A. (2005). Management of pain in older adults. Journal of the American Osteopathic Association, 105(3), 12S-17S.

Chan, S., Hadjistavropoulos, T., Williams, J., \& Lints-Martindale, A. (2014). Evidencebased development and initial validation of the pain assessment checklist for seniors with limited ability to communicate-II (PACSLAC-II). Clinical Journal of Pain, 30, 816-824.

Chen, Q., Hayman, L. L., Shmerling, R. H., Bean, J. F., \& Leveille, S. G. (2011). Characteristics of chronic pain associated with sleep difficulty in older adults: the Maintenance of Balance, Independent Living, Intellect, and Zest in the Elderly (MOBILIZE) Boston study. American Geriatrics Society, 59, 1385-1392.

Cheung, G., \& Choi, P. (2008). The use of the Pain Assessment Checklist for Seniors with Limited Ability to Communicate (PACSLAC). New Zealand Medical Journal, 121, 21-29. 
Ellis-Smith, C., Evans, C. J., Bone, A. E., Henson, L. A., Dzingina, M., Kane, P. M., Higginson, I. J., Daveson, B. A. \& BuildCARE. (2016). Measures to assess commonly experienced symptoms for people with dementia in long-term care settings: a systematic review. $B M C M e d, 14,38$.

Fine, P. G. (2011). Long-term consequences of chronic pain: mounting evidence for pain as a neurological disease and parallels with other chronic disease states. Pain Medicine, 12, 996-1004.

Fuchs-Lacelle, S. \& Hadjistavropoulos, T. (2004). Development and preliminary validation of the pain assessment checklist for seniors with limited ability to communicate (PACSLAC). Pain Management Nursing, 5, 37-49.

Greenberg, E. N. (2012). The consequences of chronic pain. Journal of Pain \& Palliative Care Pharmacotherapy, 26, 64-67.

Hadjistavropoulos, T., Herr, K., Prkachin, K., Craig, K. D., Gibson, S.J., Lukas, A., \& Smith, J. H. (2014). Pain assessment in elderly adults with dementia. Lancet Neurology, 13, 1216-1227.

Hébert, R., Desrosiers, J., Dubuc, N., Tousignant, M., Guilbeault, J., \& Pinsonnault, E. (2003). Le système de mesure de l'autonomie fonctionnelle (SMAF). La Revue de Gériatrie, 28, 323-336.

Herr, K., Bjoro, K., \& Decker, S. (2006). Tools for assessment of pain in nonverbal older adults with dementia: a state-of-the-science review. Journal of Pain and Symptom Management, 31, 170-192. 
Herr, K., Bursch, H., Ersek, M., Miller, L.L. et Swafford, K. (2010). Use of painbehavioral assessments tools in the nursing home: expert consensus recommendations for practice. Journal of Gerontological Nursing, 36, 18-29.

Herr, K., Decker, S., \& Bjoro, K. (2004). The Pain Assessment Scale for Seniors with Severe Dementia (PACSLAC). Retrieved April 19, 2016 from: http://www.docstoc.com/docs/25727431/ Tool-The-Pain-Assessment-Scale-forSeniors-with-Severe.

Hinkle, D. E., Wiersma, W., Jurs, S. G. (2003). Applied Statistics for the Behavioral Sciences, $5^{\text {th }}$ ed. Boston: Houghton Mifflin.

Hurley, A. C., Volicer, B. J., Hanrahan, P. A., Houde, S., \& Volicer, L. (1992). Assessment of discomfort in advanced Alzheimer patients. Research in Nursing \& Health, 15, 369-377.

Jensen-Dahm, C., Werner, M. U., Dahl, J. B., Jensen, T. S., Ballegaard, M., Hejl, A. M., \& Waldemar, G. (2014). Quantitative sensory testing and pain tolerance in patients with mild to moderate Alzheimer disease compared to healthy control subjects. Pain, 155, 1439-1445.

Kaasalainen, S., Akhtar-Danesh, N., Hadjistavropoulos, T., Zwakhalen, S., \& Verreault, R. (2013). A comparison between behavioral and verbal report pain assessment tools for use with residents in long term care. Pain Management Nursing, 14, e106-e114.

Katz, M. J., Lipton, R. B., Hall, C. B., Zimmerman, M. E., Sanders, A. E., Verghese, J., \& Derby, C. A. (2012). Age-specific and sex-specific prevalence and incidence of mild cognitive impairment, dementia, and Alzheimer dementia in blacks and 
whites: a report from the Einstein Aging Study. Alzheimer Disease and Associated Disorders, 26, 335-343.

Kunz, M., Mylius, V., Scharmann, S., Schepelman, K., \& Lautenbacher, S. (2009). Influence of dementia on multiple components of pain. European Journal of Pain, $13,317-325$.

Leveille, S. G., Bean, J., Ngo, L., McMullen, W., \& Guralnik, J. M. (2007). The pathway from musculoskeletal pain to mobility difficulty in older disabled women. Pain, $128,69-77$.

Lints-Martindale, A. C., Hadjistavropoulos, T., Lix, L. M., \& Thrope, L. (2012). A comparative investigation of observational pain assessment tools for older adults with dementia. Clinical Journal of Pain, 28, 226-237.

Liu, J. Y. W., Briggs, M., \& Closs, J. (2010). The psychometric qualities of four observational pain tools (OPTs) for the assessment of pain in elderly people with osteoarthritic pain. Journal of Pain and Symptom Management, 40, 582-598.

Liu, J. Y. \& Leung, D. Y. (2016). Pain treatments for nursing home residents with advanced dementia and substantial impaired communication: a cross-sectional analysis at baseline of a cluster randomized controlled trial. Pain Medicine, pii: pnw242.

Mukaka, M. M. (2012). Statistics Corner: A guide to appropriate use of Correlation coefficient in medical research. Malawi Medical Journal, 24(3), 69-71.

Muller, S., Thomas, E., \& Peat, G. (2012). The effect of changes in lower limb pain on the rate of progression of locomotor disability in middle and old age: evidence from the NorStOP cohort with 6-year follow-up. Pain, 153, 952-959. 
Mathillas, J., Lovheim, H., \& Gustafson, Y. (2011). Increasing prevalence of dementia among very old people. Age Ageing, 40, 243-249.

Organisation mondiale de la santé. (2012). La démence. Retrieved June, 2015 from: http://www.who.int/mediacentre/factsheets/fs362/fr/.

Scherder, E., Herr, K., Pickering, G., Gibson, S., Benedetti, F., \& Lautenbacher, S. (2009). Pain in dementia. Pain, 145, 276-278.

Scherder, E., Oosterman, J., Swaab, D., Herr, K., Ooms, M., Ribbe, M., Sergeant, J., Pickering, G., \& Benedetti, F. (2005). Recent developments in pain in dementia. BMJ, 330(7489), 461-464.

Shega, J. W., Hougham, G. W., Stocking, C. B., Cox-Hayley, D. S., \& Greg. A. (2004). Pain in community-dwelling persons with dementia: frequency, intensity, and congruence between patient and caregiver report. Journal of Pain and Symptom Management, 28, 585-92.

SMAF Procedure. (2013). Iso-SMAF Profiles. Retrieved from http://www.demarchesmaf.com/fr/outils/profils_iso_smaf/. Accessed November 21, 2016.

Storti, M., Bertozzo, E., Dal Santo, P., Mari, M., Dal Cengio, L., Forni, C., Giantin, V., \& Zucchi, A. (2014). The measurement of pain in patients with dementia: a review of the most appropriate tools. Recenti Progressi in Medicina, 105, 166-174.

Stubbs, B., Patchay, S., Soundy, A., \& Schofield, P. (2014). The avoidance of activities due to fear of falling contributes to sedentary behavior among communitydwelling older adults with chronic musculoskeletal pain: a multisite observational study. Pain Medicine, 15, 1861-1871. 
Takai, Y., Yamamoto-Mitani, N., Okamoto, Y., Koyama, K., \& Honda, A. (2010). Literature review of pain prevalence among older residents of nursing homes. Pain Management Nursing, 11, 209-223.

Warden, V., Hurley, A. C., \& Volicer, L. (2003). Development and psychometric evaluation of the Pain Assessment in Advanced Dementia (PAINAD) scale. Journal of the American Medical Directors Association, 4, 9-15.

Wary, B., Serbouti, S., \& Doloplus, C. D. (2001). Validation d'une echelle d'evaluation comportementale de la douleur chez la personne agee. Douleurs, 2, 35-38.

Zwakhalen, S. M., Hamers, J. P., Abu-Saad, H. H., \& Berger, M. P. (2006a). Pain in elderly people with severe dementia: a systematic review of behavioural pain assessment tools. BMC Geriatrics, 6, 3.

Zwakhalen, S. M., Hamers, J. P., \& Berger, M. P. (2006b). The psychometric quality and clinical usefulness of three pain assessment tools for elderly people with dementia. Pain, 126, 210-220.

Zwakhalen, S. M., van der Steen, J. T., \& Najim, M. D. (2012). Which score most likely represents pain on the observational PAINAD pain scale for patients with dementia? Journal of the American Medical Directors Association, 13, 384-389. 


\section{FIGURE LEGENDS}

Figure 1. Participant flow chart

Figure 2. Scoring times for the three assessment scales (mean \pm SD). PACSLAC scoring time was higher than the PACSLAC-II, which was higher than the PAINAD $(* * * \mathrm{p}<$ $0.001)$. 
Figure 1

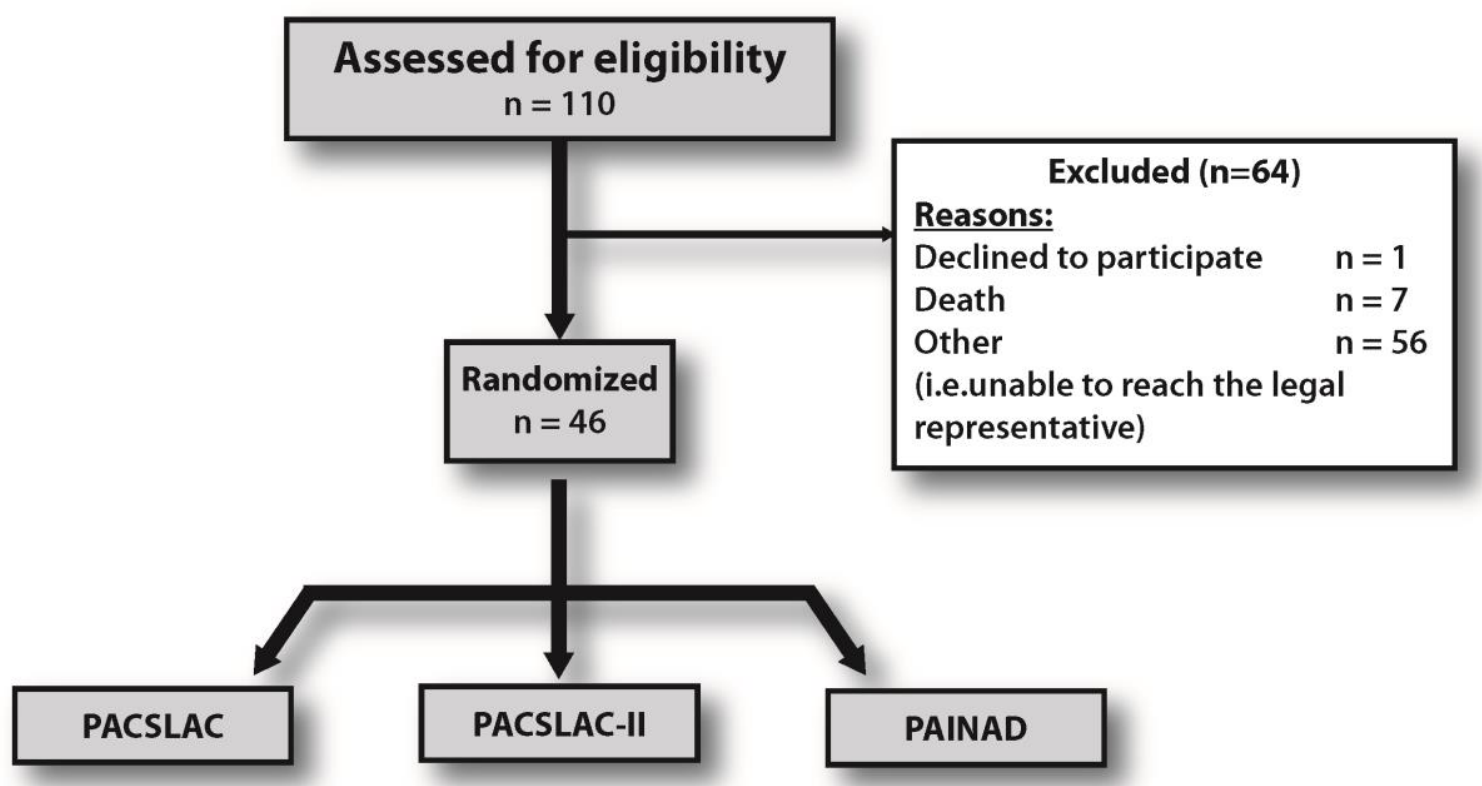


Figure 2

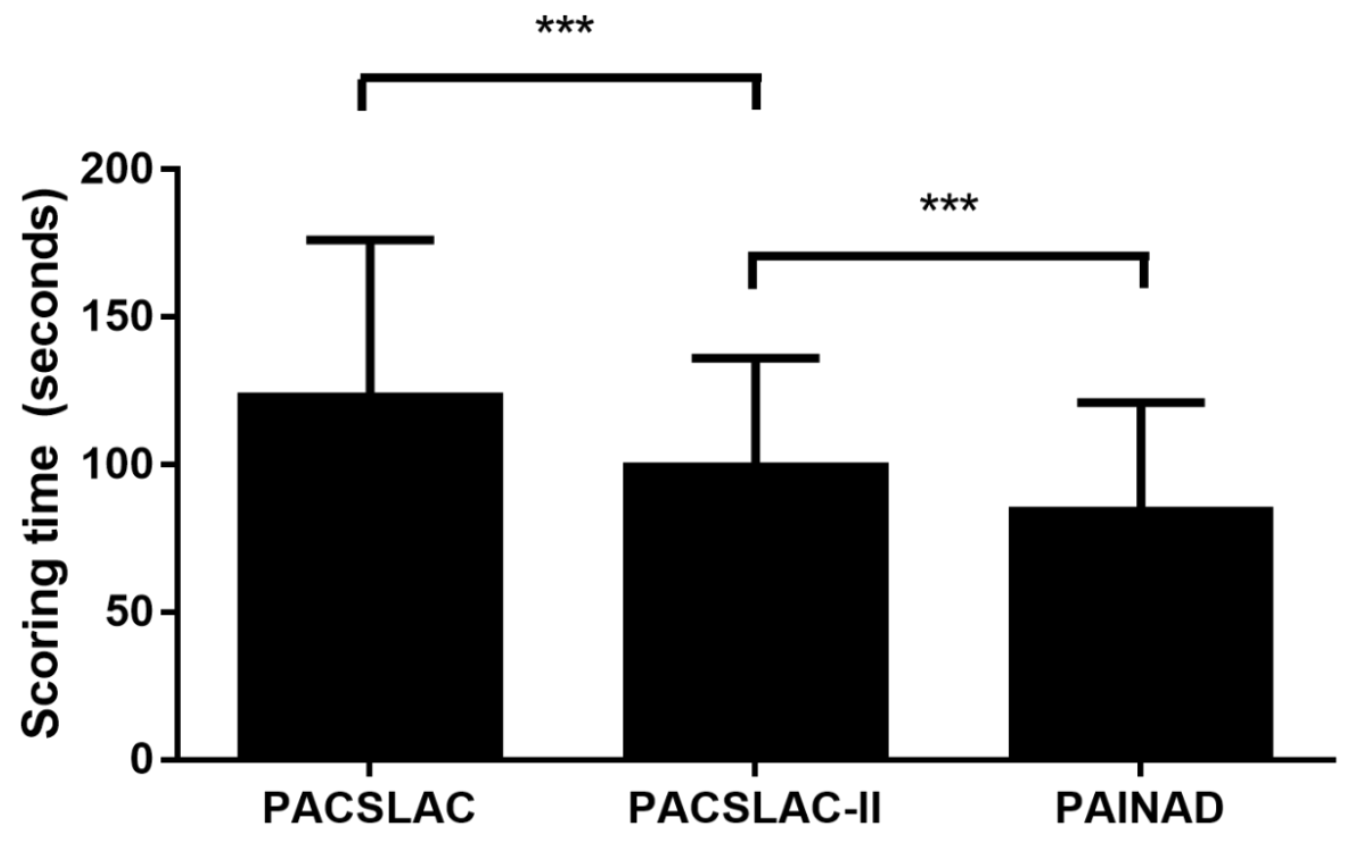


Table 1: Sociodemographic profile of participants $(n=46)$

\begin{tabular}{ll}
\hline Sociodemographic characteristics & $\mathrm{n}(\%)$
\end{tabular}

\begin{tabular}{ll}
\hline Gender (women) & $36(78.3)$
\end{tabular}

$\begin{array}{ll}\text { Age (years) (mean } \pm \text { SD) } & 83 \pm 10\end{array}$

\section{Category 3: profiles 8 and 10}

Iso-SMAF profiles*

$43(93.5)$

Category 4: profiles 1112,13 and 14

\footnotetext{
* The Iso-SMAF profiles allow the classification of elders per the intensity and type of service needed to maintain their autonomy (SMAF Procedure, 2013). The 14 Iso-SMAF profiles are generally grouped into 4 categories. Category 3 corresponds to elders having predominant loss in cognitive functions, whereas category 4 includes elders with serious mixed (mobility and cognitive) alterations.
} 
Table 2: Correlations between the PACSLAC-II, the PACSLAC and the PAINAD

\begin{tabular}{lllc}
\hline Pain Questionnaires & PACSLAC & PACSLAC-II & PAINAD \\
\hline PACSLAC & 1 & 0.613 & $0.625^{*}$ \\
PACSLAC II & $0.613^{*}$ & 1 & $0.645^{*}$ \\
PACSLAC (Facial expressions) & $0.547^{*}$ & $0.795^{*}$ & $0.479^{*}$ \\
PACSLAC (Body movements + Facial expressions) & $0.565^{*}$ & $0.963 *$ & - \\
PAINAD & $0.625^{*}$ & 0.645 & 1 \\
\hline
\end{tabular}

Statistical analysis: Pearson Rho

* Statistically significant: $\mathrm{p} \leq 0.001$ 